\title{
Laser-Based Fabrication of Nanofoam inside a Hollow Capillary
}

\author{
Alexander F. Courtier, James A. Grant-Jacob, Rand Ismaeel, Daniel J. Heath, Gilberto Brambilla, \\ William J. Stewart, Robert W. Eason, Ben Mills*
}

Optoelectronics Research Centre, University of Southampton, Southampton, UK

Email: ^bm602@orc.soton.ac.uk

How to cite this paper: Courtier, A.F., Grant-Jacob, J.A., Ismaeel, R., Heath, D.J., Brambilla, G., Stewart, W.J., Eason, R.W. and Mills, B. (2017) Laser-Based Fabrication of Nanofoam inside a Hollow Capillary. Materials Sciences and Applications, 8, 829-837.

https://doi.org/10.4236/msa.2017.812060

Received: September 14, 2017

Accepted: October 31, 2017

Published: November 3, 2017

Copyright $\odot 2017$ by authors and Scientific Research Publishing Inc. This work is licensed under the Creative Commons Attribution International License (CC BY 4.0).

http://creativecommons.org/licenses/by/4.0/

\begin{abstract}
Highly porous nanofoam can be fabricated via multiphoton ablation of a material by raster-scanning femtosecond laser pulses over the material surface. Here, we show the fabrication of nanofoam on the inside surface of a hollow silica capillary that has an inner and outer diameter of 640 and $700 \mu \mathrm{m}$ respectively. A thin layer of nanofoam was fabricated over $\sim 70 \%$ of the inner surface of the capillary. Ray-tracing simulations were used to determine the positional corrections required to account for refraction on the curved surface and also to explain the inability to fabricate nanofoam on the side walls of the capillary.
\end{abstract}

\section{Keywords}

Laser Ablation, Multiphoton Ablation, Capillary, Glass, Fabrication, Nanofoam

\section{Introduction}

Nanofoams are a class of nanostructured porous materials, and have potentially desirable applications in photonics, electronics, chemistry and medicine [1]-[6]. Here, we use femtosecond laser pulses as the fabrication tool, as they offer the potential for precise control of the location where the nanofoam is produced. The fabrication of nanofoam via high repetition rate laser ablation has previously been demonstrated in many materials, including carbon [7], silica glass [8], silicon [9] and chalcogenide glass [10]. In practice, the fabrication requires raster-scanning a laser focus over a target material, with a carefully chosen separation between adjacent raster-scanned lines.

The fabrication process when using high-repetition-rate femtosecond pulses, is understood as follows. The energy from the laser pulse is absorbed at the sur- 
face, and transferred into lattice vibrations (i.e. heat) on a picosecond and longer timescale, leading to a significant accumulation of energy at the location of the laser focus, causing the material to melt and form a plasma. If the time separation between laser pulses is shorter than the glass melt time, the melted region is prevented from solidifying. As the energy increases further, jets of high temperature material are forced out of the plasma and subsequently cool rapidly, forming nanowires whose composition reflects that of the target material [8]. The result of this process is a highly disordered mesh of nanowires, which we refer to here as nanofoam.

This work builds on the study of the fabrication of nanofoam on planar surfaces [11], such as glass slides, and translates this approach to fabrication inside a hollow glass capillary. Experimental results showing the fabrication of nanofoam inside a hollow capillary are presented, assisted via the use of ray-tracing simulation that describes how to correct for the effects of refraction of light from the curved surfaces. This proof-of-principle result may facilitate a diverse range of end applications, for example, microstructured optical fibres and particle filtration devices.

In order to fabricate nanofoam inside a hollow capillary, we require the laser light to propagate through the air-glass interface at the outer surface, and be absorbed at the glass-air interface at the inner surface. To achieve this, we must take advantage of multiphoton processes during fabrication, which can enable very short pulses of light to pass through a material when unfocussed, as the peak intensity is too low, whilst being absorbed by the material at the focus. Therefore, femtosecond laser pulses were used for this approach.

In this manuscript, Section 2 illustrates the experimental setup and the fabrication process, while Section 3 provides a theoretical discussion on the refraction from the curved surface of a hollow capillary and an approach for compensating for the resultant positional errors. The experimental results are discussed in Section 4, and conclusions are presented in Section 5.

\section{Experimental Setup}

Laser pulses from a Ti: sapphire amplifier ( $150 \mathrm{fs}$ pulse duration, $800 \mathrm{~nm}$ central wavelength), operating at a repetition rate of $250 \mathrm{kHz}$, were focused using a $50 \mathrm{x}$ objective (Nikon LU Plan, NA = 0.55), onto the inner surface of a pure silica, hollow capillary. The focused spot size, which was approximately Gaussian in profile, without any refraction effects was $\sim 3 \mu \mathrm{m}$ diameter, with associated single pulse fluence $\sim 56 \mathrm{~J} / \mathrm{cm}^{2}$. However, due to refraction effects (as illustrated by the simulations in Section 3) these values represent a lower limit for the spot size, and an upper limit for the fluence. The capillary had an outer diameter of 700 $\mu \mathrm{m}$, inner diameter $640 \mu \mathrm{m}$, and wall thickness of $30 \mu \mathrm{m}$. The laser was scanned back and forth in straight lines that follow the internal surface of the hollow capillary, via XYZ positional stages on the sample. The chosen raster-scanning geometry is shown in Figure 1. A total of 400 lines (illustrated by red lines along 


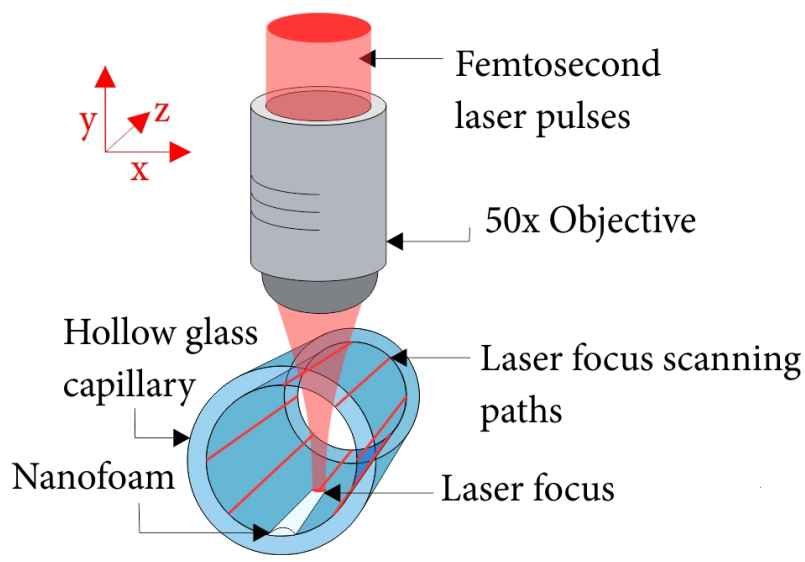

Figure 1. Schematic of experimental setup for the fabrication of nanofoam inside a hollow capillary, where $\mathrm{Z}$ axis is defined as along the length of the capillary.

the length of the inside of the capillary in the figure) were scanned, separated from an adjacent line by $\sim 5 \mathrm{~m}$ along the curve of the capillary and each with a scan speed of $3 \mathrm{~mm} / \mathrm{s}$, where both values were found optimal for maximum volume production for this setup when fabricating nanofoam on a planar substrate.

The objective here was to maximize the amount of nanofoam on the inside surface. Whilst rotation of the capillary could be possible in principle, here we explore the feasibility of the approach of only moving the capillary in XYZ, and hence with no rotation, for both simplicity and accuracy. Rotation stages require an exact center of rotation to be defined, and as such, would require precise positioning of the capillary with respect to the center of movement. Previous work has shown that for planar substrates, the top $\sim 1 \mu \mathrm{m}$ of the sample is sacrificed, and turned into nanofoam with depth up to $100 \mu \mathrm{m}$, and with a density as low as $1 \%$ of the original material. The amount of nanofoam produced and the appearance strongly depends on the material type and the experimental parameters.

\section{Refraction Effects from a Hollow Capillary}

This section provides a theoretical discussion of refraction effects that must be taken into account when trying to focus light on the inside surface of a hollow capillary, where the capillary can only be translated in XYZ and not rotated, and where the light focus position is static. The theoretical results in this section illustrate the positional corrections that were used in Section 4 in order to compensate for refractive effects, and hence ensure that the laser focus was scanned along the inner surface of the capillary. This section also presents a qualitative description of the limitation of this approach, in terms of the possible range of angles that can be utilized for nanofoam fabrication. As shown in Figure 1, the Z axis is defined as along the length of the capillary, and hence the effects of refraction are independent of the $\mathrm{Z}$ position. Therefore, the simulations are only for a single XY plane. The motivation behind investigating this configuration is 
that it matches a laser machining setup that does not include a rotation stage. To quantify these effects, in this section we discuss a simulation framework, based on ray-tracing, which provides an understanding of the limitations of this laser fabrication approach, along with specific positional corrections. The size scales for the inner and outer diameters $(640 \mu \mathrm{m}$ and $700 \mu \mathrm{m})$, the numerical aperture of the objective $(\mathrm{NA}=0.55)$ and the capillary refractive index $(\mathrm{n}=1.45)$, are chosen here to match the experimental results in Section 2.

Geometrical ray-tracing can be used to characterize the effects of refraction caused by propagation through a hollow capillary. For the simulation, 15 rays, at 5 degree spacing between -35 degrees and +35 degrees, were propagated through a $2 \mathrm{D}$ array of zeros and ones, corresponding to the presence of air $(\mathrm{n}=1)$ and glass capillary $(\mathrm{n}=1.45)$ respectively. The propagation of each ray was calculated analytically taking into account the angle of the interfaces and the change in refractive index at each position, and the size of the beam at all positions was calculated by taking the standard deviation of the separation of the rays (in the direction perpendicular to the propagation direction), where the minimum value of this standard deviation of separations corresponded to the resultant focus position. In general, refraction at an interface caused the rays to diverge from their original direction, hence resulting in a larger minimum separation that also occurred in a different position to that if there was no capillary present. Each simulation trial provided: 1) the XY positions of the focus if there was no capillary, referred to as the "stage position"; 2) the XY position of the focus modified due to refraction, referred to as the "focus position"; and 3) the size of the focus due to refraction, referred to as the "focus size". To obtain this dataset, the focus position and focus size were recorded for a set of stage positions, hence providing a mapping between the stage position and the resultant focus position and focus size.

The challenges of focusing at every position on the inside surface of a hollow capillary is illustrated in Figure 2, which shows the highest possible fluence, at all possible focus positions across the capillary cross-section, as a percentage of the incident light fluence. Foci that were outside the outer diameter of the capillary have been removed from the figure for clarity. Regions inside the capillary cross-section that are black (i.e. 0\%), for example on the side walls, are positions that are theoretically impossible to focus on. A value of 25 represents a fluence that is $25 \%$ of the incident light fluence. As shown in the figure, the bottom and top regions experience a high maximum fluence, whilst regions on the side generally experience only a low maximum fluence. As the nanofoam fabrication process is extremely dependent on fluence, it is clear from this figure that some regions of the inside surface will correspond to larger volumes of fabricated nanofoam.

As discussed earlier, the ray-tracing simulations provide a mapping from the stage position (i.e. the physical position of the stage) to the focus position (i.e. the resultant position of the focus). By reversing this mapping, the required stage 


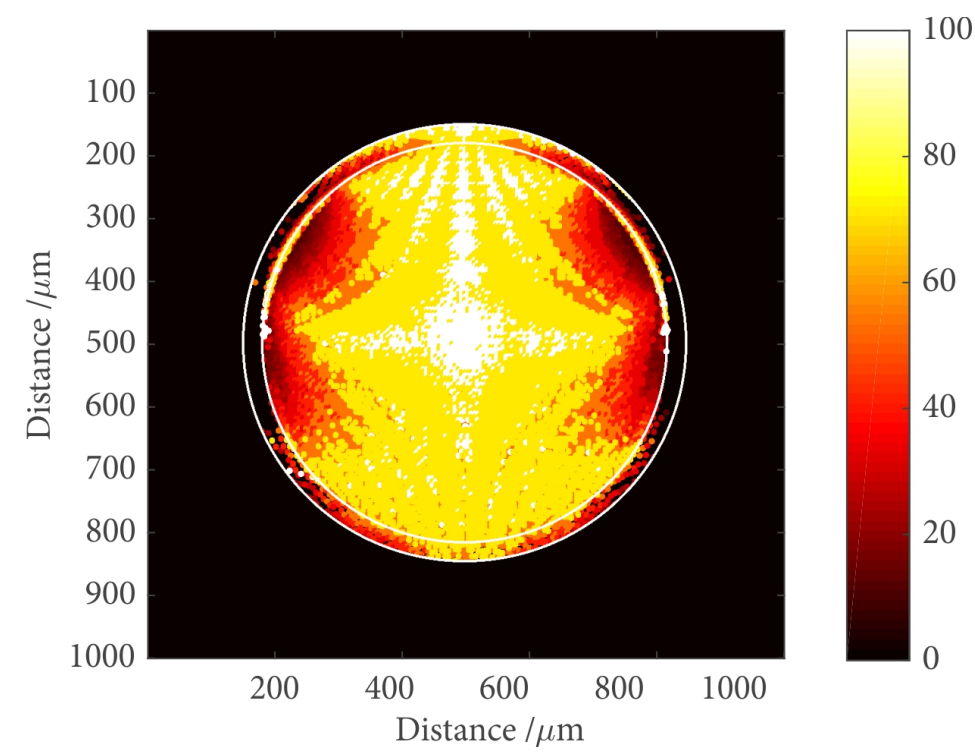

Figure 2. Maximum fluence possible at each position across the cross-section of the capillary, as a percentage of the incident fluence. The large white circles correspond to the inner and outer capillary diameter.

position for any possible focus position can be determined. This result is shown in Figure 3, which describes the positional corrections, at a series of locations, that must be applied in order to focus on the inside surface of the capillary. The two large green circles correspond to the inner and outer diameter of the capillary. The figure shows stage positions (red circles) that results in focus positions (blue circles) that coincide with the inner surface, and hence the difference between each pair is the required positional correction. Not all desired focus positions are possible (according to the simulations), and hence the result is that the figure does not provide the corrections for some positions on the sides of the capillary.

\section{Results and Discussion}

In this section, experimental results showing the fabrication of nanofoam are presented. The laser focus was scanned starting on the bottom inside surface, up one side, then the other side, then finally the top, to ensure that the laser light would not have to propagate through the fabricated nanofoam at any point, which would have introduced additional distortions. The objective here was to develop a method for coating the maximum amount of the inside of the capillary in nanofoam.

Since optical microscopes lacked the resolution to image the nanofoam and suffer from refractive distortions similar to those discussed in Section 3, and since scanning electron microcopy (SEM) only allows imaging the surface of materials, nanofoam was generated along the last $\sim 1 \mathrm{~mm}$ of the hollow capillary, to allow observation of nanofoam at the end-face of the capillary using a SEM. Figure 4 shows an SEM image of the cross-section of a hollow capillary, where 


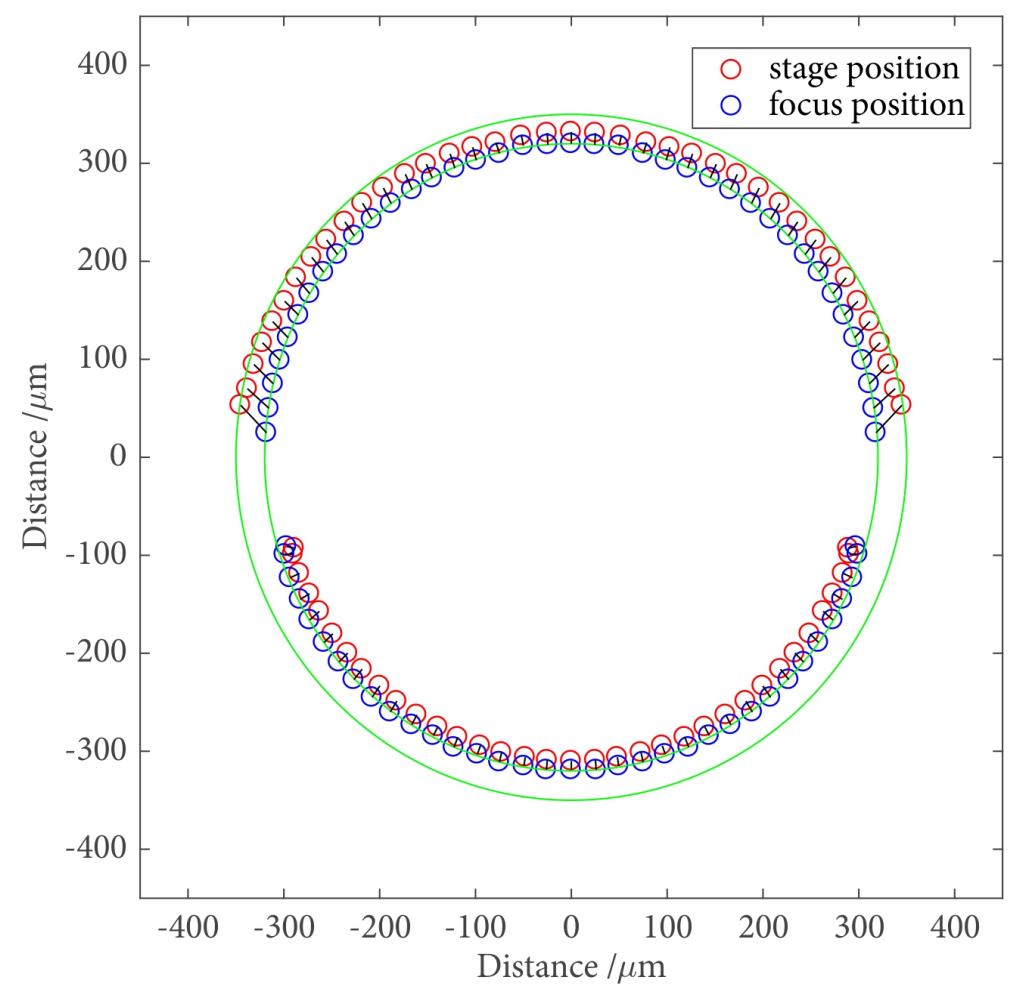

Figure 3. Positional corrections, for a range of position, when focusing on the inside surface of a hollow capillary, as a consequence of refraction. The green circles correspond to the inner and outer diameter of the capillary.

nanofoam has been fabricated along the nearest $\sim 1 \mathrm{~mm}$ of the inside of the capillary.

Analysis of the SEM images show that nanofoam has been fabricated for approximately \pm 40 degrees on the top surface, \pm 80 degrees on bottom surface, and hence leaving $\sim 60$ degrees uncoated on each side (a total of $\sim 70 \%$ coverage therefore). As shown in Figure 4, a small "flake" of nanofoam is present on one side of the inner surface, which we believe had been dislodged from the upper surface. As discussed in Section 3, calculations indicate that some positions are not possible to form nanofoam, particularly the side walls, and this is evident in the experimental data. The nanofoam itself is not formed to the same extent across the internal surface, and some regions have up to ten times the height compared to other adjacent positions. We attribute this to a combination of the following three reasons. Firstly, any topological imperfections in the capillary, including damage and debris, would result in refractive effects leading to unintended changes to the focus size and position, hence modifying the total volume of nanofoam that was fabricated at that specific position. Secondly, the angular distribution (with respect to the direction of the incident laser pulses) of the high temperature jets of material that form the nanofoam is not known. For a planar substrate, the particular distribution is not important, as the effect is averaged out. For the case presented here, the distribution is extremely important, as the angle of the surface with respect to the direction of the incident laser pulses is 


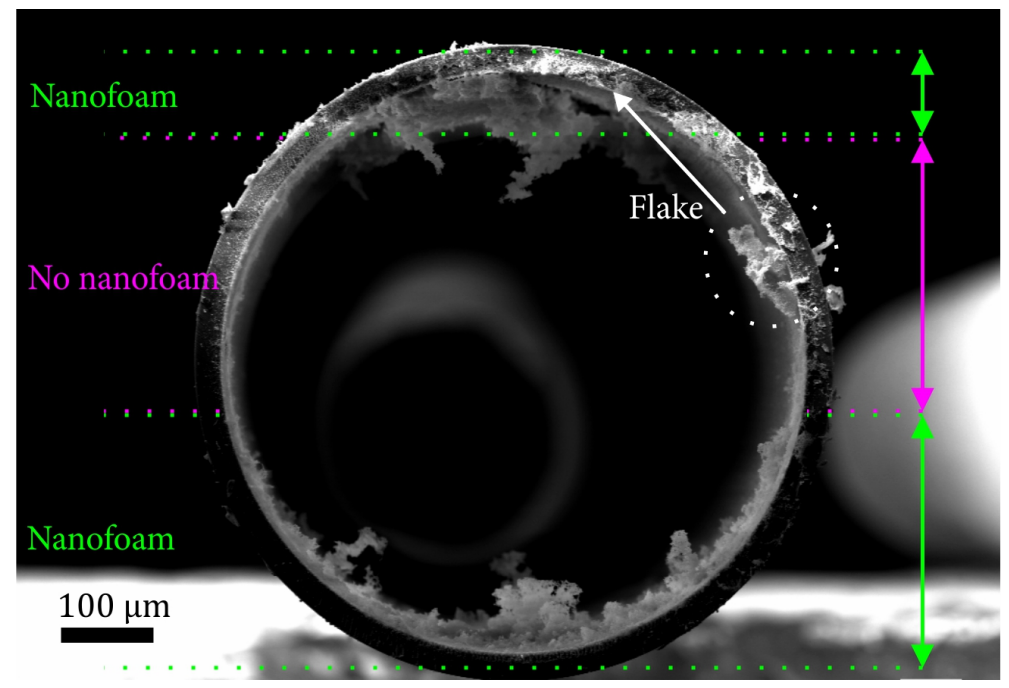

Figure 4. SEM image of nanofoam that has been fabricated along the inside of a hollow capillary, for the $\sim 1 \mathrm{~mm}$ nearest to the viewer. The laser pulse was incident from the top of the capillary in this orientation during fabrication. The dashed circle shows the location of a flake that has been displaced.

not constant. For example, if the volume of material ejected in the direction perpendicular to the incident laser pulses is different to that ejected from the parallel direction, the volume of fabricated nanofoam will not be constant over all positions on the inner surface. This effect could be compounded due to the plasma being constrained within a restricted geometry. Thirdly, and probably most significant, the result shows the need for a more sophisticated understanding of the refractive effects. The positional offsets were determined by attempting to correct the effects of refraction. Whilst these corrections enable the focus to follow the inner surface, the size of the focus was not taken into account. For example, towards the sides, even though the focus is positioned on the inner surface, the focus size is much larger and the intensity is therefore reduced. This has two direct consequences, namely that the intensity is reduced resulting in a lower volume of nanofoam, and secondly, the spatial overlap of the pulses is now changed from the optimal values of a $\sim 3 \mu \mathrm{m}$ diameter beam separated by $\sim 5 \mu \mathrm{m}$. As shown in previous work [11], changes to the spatial overlap have significant effects on the volume of nanofoam produced.

Figure 5 shows some regions of the nanofoam fabricated in Figure 4 at a higher imaging magnification, to illustrate the intricate structure that forms the basis for the nanofoam fabricated by this method. As shown in the figure, the interconnected nanowires that form the nanofoam are typically of diameter 200 $\mathrm{nm}$ and smaller. The fabricated nanofoam has considerable variation in terms of its structure and overall shape, for example, Part d) shows a region of nanofoam with a height of $\sim 150 \mu \mathrm{m}$.

\section{Conclusion}

In conclusion, we have demonstrated the laser-based fabrication of nanofoam 

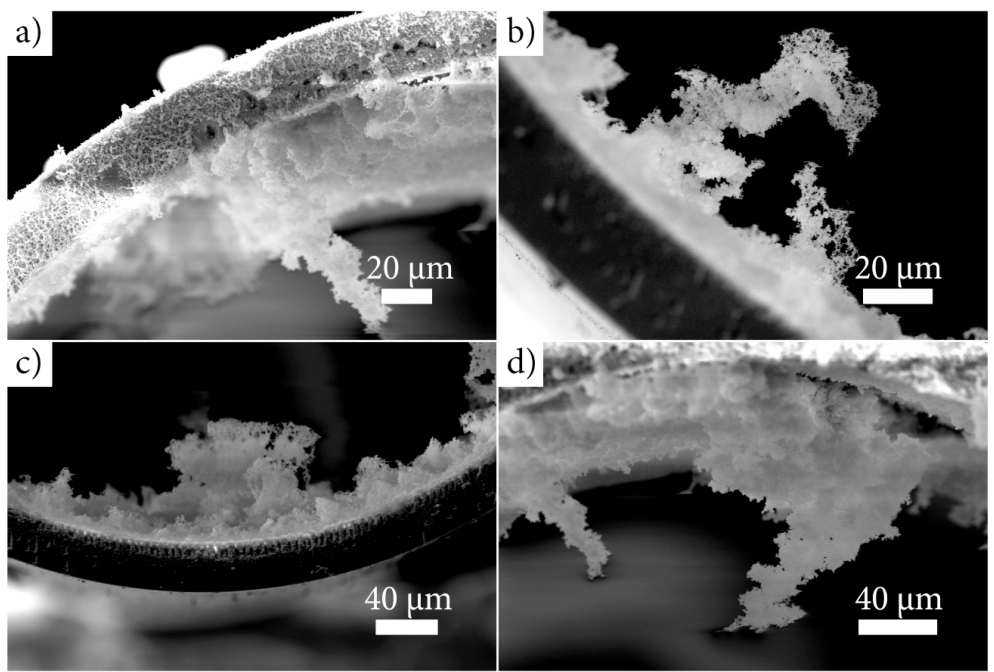

d)

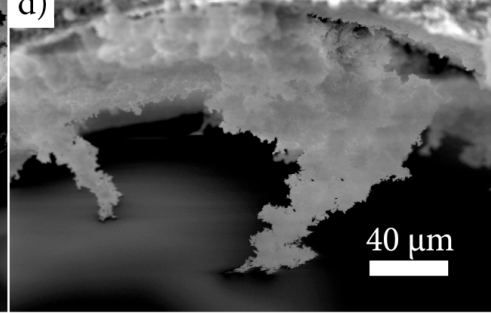

Figure 5. SEM images that highlight the intrictate structures that form the basis for fabricated nanofoam.

inside a hollow glass capillary, by taking into account positional corrections that occur as a consequence of refraction of the laser pulses at the curved surfaces. Coverage of $\sim 70 \%$ of the inside of the inner surface of a capillary was achieved. The results show that further refinement of this technique is needed in order to increase the total percentage fill of nanofoam produced. This could be achieved, for example, through the use of smaller capillaries, a higher laser fluence, or via multiple laser beams. This work may have potential across a wide range of applications, such as microstructured optical fibres and filtration devices.

\section{Acknowledgements}

This work was supported by the UK Engineering and Physical Sciences Research Council (EPSRC) Grant Number EP/N03368X/1. The data for this work are accessible through the University of Southampton Institutional Research Repository (DOI:10.5258/SOTON/D0247).

\section{References}

[1] Brock, S.L. (2007) Materials Science. Filling a Void. Science, 317, 460-461. https://doi.org/10.1126/science.1146517

[2] Du, A., Zhou, B., Li, Y., Li, X., Ye, J., Li, L., Zhang, Z., Gao, G. and Shen, J. (2011) Aerogel: A Potential Three-Dimensional Nanoporous Filler for Resins. Journal of Reinforced Plastics and Composites, 30, 912-921. https://doi.org/10.1177/0731684411407948

[3] Bag, S., Trikalitis, P.N., Chupas, P.J., Armatas, G.S. and Kanatzidis, M.G. (2007) Porous Semiconducting Gels and Aerogels from Chalcogenide Clusters. Science, 317, 490-493. https://doi.org/10.1126/science.1142535

[4] Sun, H., Xu, Z. and Gao, C. (2013) Multifunctional, Ultra-Flyweight, Synergistically Assembled Carbon Aerogels. Advanced Materials, 25, 2554-2560.

https://doi.org/10.1002/adma.201204576

[5] Job, N., Thery, A., Pirard, R., Marien, J., Kocon, L., Rouzaud, J.-N., Beguin, F. and 
Pirard, J.-P. (2005) Carbon Aerogels, Cryogels and Xerogels: Influence of the Drying Method on the Textural Properties of Porous Carbon Materials.Carbon, 43, 2481-2194.

[6] Vainos, N.A., Karoutsos, V., Mills, B., Eason, R.W. and Prassas, M. (2016) Isotropic Contractive Scaling of Laser Written Microstructures in Vitrified Aerogels. Optical Materials Express, 612, 3814-3825.

[7] Rode, A.V., Gamaly, E.G. and Luther-Davies, B. (2000) Formation of Cluster-Assembled Carbon Nano-Foam by High-Repetition-Rate Laser Ablation. Applied Physics A, 70, 135-144. https://doi.org/10.1007/s003390050025

[8] Venkatakrishnan, K., Vipparty, D. and Tan, B. (2011) Nanofibre Fabrication by Femto-Second Laser Ablation of Silica Glass. Optics Express, 19, 15770-15776. https://doi.org/10.1364/OE.19.015770

[9] Zhang, Y.F., Tang, Y.H., Wang, N., Yu, D.P., Lee, C.S., Bello, I. and Lee, S.T. (1998) Silicon Nanowires Prepared by Laser Ablation at High Temperature. Applied Physics Letters, 72, 1835-1837. https://doi.org/10.1063/1.121199

[10] Juodkazis, S., Misawa, H., Louchev, O.A. and Kitamura, K. (2006) Femtosecond Laser Ablation of Chalcogenide Glass: Explosive Formation of Nano-Fibres against Thermo-Capillary Growth of Micro-Spheres. Nanotechnology, 17, 4802-4805. https://doi.org/10.1088/0957-4484/17/19/003

[11] Grant-Jacob, J.A., Mills, B. and Eason, R.W. (2014) Parametric Study of the Rapid Fabrication of Glass Nanofoam via Femtosecond Laser Irradiation. Journal of Physics D: Applied Physics, 47, Article ID: 5055105. https://doi.org/10.1088/0022-3727/47/5/055105 